\title{
Discussion on the Innovation of Traditional Graphic Art in the Hakka Culture
}

\author{
Wei Liü, a \\ ${ }^{1}$ Fujian Jiangxia University, Fuzhou, Fujian, China, 350001 \\ a57858152@qq.com
}

Keywords: Hakka culture; Traditional graphic; Innovation

\begin{abstract}
In the national culture, the Hakka culture is an important part of our country's pluralistic national culture, and has unique connotation and spirit. the traditional graphics as a visual element in Hakka culture has distinctive personality and unique style. Hakka traditional graphics is derived from cultural accumulation of the Hakka people in the life and practice, its humanistic value is significant. Currently, the promotion of Chinese traditional culture is the development trend. How to understand the innovation of Hakka traditional graphic art form is the important value of traditional culture inheritance in modern design and the mission of history.
\end{abstract}

\section{The Hakka traditional graphic overview}

In the national culture, Hakka culture has its unique spiritual connotation and characteristics, is an important part of our national culture. Hakka traditional pattern as a visual element is widely used in modern design, has a unique distinctive national characteristics and style. Under the background of economic and cultural globalization, it is an important responsibility to carry forward the national culture. For modern designers, how to skillfully apply the traditional graphic elements of Hakka culture to modern design is a question that every modern designer should consider, while how to combine national culture with modern life and modern art is a question that every designer needs to think about. Carrying forward the national culture is not blindly conservative and abiding by the inheritance, it is necessary to inherit but also open up the design ideas. Hakka traditional graphics have the simple style, with a wide range of expression, such as: Hakka house building decoration, Hakka traditional clothing, folk paper cutting, wood carving, stone and so on. Hakka traditional graphics also have a very obvious feature: First, Hakka traditional graphics is historic. Chinese culture is brilliant, has a long history, like a large-scale and extraordinary symphony, and Hakka culture is one of the exciting music. In the past, Hakka traditional culture created a batch of Hakka talents; now the Hakka culture, flashing the ideal of the humanities, is nurturing a new generation of Hakka children. The torch of Central Plains civilization is spreading in the displacement of the migration; burning in the wandering suffering. Hakka traditional graphics is the visual representation of Hakka culture. Second, Hakka traditional graphics are inherited. Hakka traditional culture has a long history, like a branch of green leaves of the towering trees. Growing in the Central Plains, in Heluo's ancient soil. Hakka culture is rich and colorful, simple and prudent; pragmatic spirit to avoid the virtual; The temperament against the chase, and the "native emotion" Hakka culture is worthy of our future heritage and spreading down. At the same time, Hakka culture has been weakened in the process of social-economic and cultural development to some extent, but the desire of Hakka compatriots on the heritage of Hakka culture become much stronger, therefore, Hakka traditional graphics application and innovation can provide visual presentation for the continuation of Hakka culture effectively.

\section{The significance of Hakka traditional graphic art form innovation}

Under the background of globalization, inheriting, carrying forward, developing Hakka culture, is the responsibility of every designer. Hakka culture wide and sincere, a large number of graphic patterns of Hakka modern show the far-reaching significance of Hakka culture, China is a multiethnic aggregation of the country, we only maintain the unique national culture, in order to achieve 
the world cultural diversity. Hakka occupies an irreplaceable position in our multi-ethnic culture, studying and re-designing the Hakka traditional graphics, exploring the application in modern design of Hakka traditional graphics, finding out how to show the characteristics of Hakka traditional graphics, has important significance for the inheritance and development of our culture.

National culture is profound, China has a melodious history and culture, and each national traditional culture contains many artistic atmosphere, for the designers, only applying the artistic elements in traditional culture, national culture, to the modern design, better to be understood and accepted by people, fully tap the value of the national culture and traditional culture, are the questions that the designer should pay attention to and consider. In the era of "entertainment to death", the traditional interpretation of teaching knowledge and indoctrination is difficult to attract young people's attention, especially young people's eyes, let alone understanding and loving, so we can not use old eyes and old way to education the young people how to understand and focus on Hakka traditional graphics culture. We should actively keep up with the pace of the times, taking the Hakka traditional graphics culture to the times, to allow more young people to understand what is Hakka traditional graphics culture, and the importance of protection of Hakka traditional graphic culture. Only do like that, the culture of Hakka traditional graphics can be better inherited and protected in the new generation of young people.

\section{The methods on the Hakka traditional graphic art form innovation}

Art innovation is based on the original artistic creation and works of art, to change the original form of artistic creation on the basis of the current stage of artistic atmosphere and artistic expression, and form new artistic creation and modern creative approach. On the Hakka traditional graphic art form of innovation, we should strive to explore new artistic creation methods and models, rather than blindly abide by the old visual form. There are no two identical leaves in the world, nor two identical sand, and art innovation as well. Hakka traditional graphic art form innovation is not the traditionally mechanically formed, nor the one-sided representation of the heritage, should retain their own cultural characteristics on the basis of bold and innovative expression, should excavate available Hakka culture traditional elements for modern design, such as a simple tradition graphic style, to know its origin, the implied meaning, abstractly summary on the basis of them, once refining processing again, from the inheritance and development of the expression form and the depth of the inherent spirit to find different media to achieve.

With the development of domestic design and creative industries, the "Chinese style" and "national wind" have been quietly rising. The designs with the characteristics of Hakka culture has gradually emerged, with the foresight of the designers, mining the use of Hakka culture unique graphics, combining with modern design, not only open up new ideas for the design, but also promote the Hakka culture and traditional culture. Designers boldly and innovatively integrate the unique culture of Hakka culture into modern design works. They have obtained more excellent results in the design of various fields In addition to the direction of visual communication, ring art design field on how to apply Hakka culture graphics to the design also has a good show. Like that the designer boldly using Hakka culture graphics, patterns to integrate them into the modern home design. Currently, Hakka lifestyle gradually subsided, in the background of urbanization and localization of Hakka cultural areas, heritage to protect the traditional culture, in the modern life they did not forget to promote the Hakka culture. Advertising, posters, packaging, interactive, ring art, handicrafts and many other design categories, there are numerous fine design works using Hakka culture and graphic design.

At the same time, using new technology and new media in all aspects and direction, applying the transmission system of three-dimensional model, to establish the unique system of Hakka traditional graphic culture. Through the current popular Internet communication model to carry out a new round of communication. Through network media resources with high user attention, such as micro blog, blog and other communication channels to widely disseminate. Besides, it is necessary to attach the attention of website with discourse right, such as the local government website, especially websites having established Hakka traditional culture, through spread so that the public 
can be aware of the importance of Hakka traditional graphics culture. So that the public can actively participate in Hakka traditional graphic culture protection.

Artistic innovators are more likely to extract unique elements in Hakka's traditional graphics, through digital and new media technology, it can also set up film and television works. To reflect its creative process in the dissemination process can display the core values of Hakka traditional elements graphics completely, and can further reflect the Hakka traditional elements graphics results, conducive to interact with the masses, and actively develop the cultural and creative industries.

\section{Conclusion}

Based on the Hakka culture and the Hakka traditional graphic features, this paper fully deduces valuable artistic elements, through combining with modern modernity to show the artistic value of Hakka traditional graphics and the connotation of Hakka culture. Besides, it summarized related modern design forms and the importance of inheritance. Under the background of globalization, inheritance and development of Hakka traditional culture, in-depth understanding graphic meaning of the Hakka culture, making the design to enhance the national pride of is the responsibility of each designer. China need to develop their own artistic innovation model, cultivate outstanding artistic professionals, establishing China's unique artistic innovation thinking and artistic innovation works, only in the way, can we establish a rich cultural system with Chinese characteristics for China's socialist cultural construction, and paste the unique label of China on them. Hakka culture is wide and sincere, especially the graphics with Hakka traditional culture all show the essence and farreaching significance of Hakka culture, Hakka traditional culture is actually worth a lot of research and development in some aspects, China is country with a multi-ethnic aggregation, only maintaining the uniqueness of the traditional culture, can we keep the diversity of the world's culture.

\section{Acknowledgements}

Fund Project: 2014 Fujian Young Teacher Education Research Project "Creative Brand Research in the Perspective of Fujian and Taiwan Traditional Culture", Item Number: JBS14217.

\section{References}

[1] Chen Yuanshuang. Integration of Tradition and Modernity - Research on the Development of Modern Pottery in the View of Hakka Culture in Luodai Town [J] .Ceramic Art Technology. 2015, 7

[2] Liu Hefu. Where does the guest come from? - the origin and charm of Hakka culture [J]. Culture. 2014, 1

[3] Bao Xiaoli, Xu Lianghui. Cultural Heritage Tourism and Hakka Culture Inheritance and Innovation in the context of urbanization - taking Fujian province Yongding Tulou as an Example [J]. Journal of Cultural Studies. 2015

[4] Xu Weiqun. The symbolic analysis of Hakka cultural resources and its innovative significance [J]. Journal of Longyan University. 2014, 12

[5] Lan Qifa. Taiwan Hakka cultural heritage and its enlightenment [J]. Journal of Jimei University (Philosophy and Social Edition). 2015, 1

[6] Li Song Xu Weiqun. Inheritance and Utilization of Hakka Intangible Cultural Heritage from the Perspective of Cultural Symbols - Taking Hakka in Western Fujian province as an Example [J]. Journal of East China University of Science and Technology (Natural Science Edition). 2015, 6

[7] Niu Meng. The visual performance of Folk pattern in the contemporary design [D]. Dalian University of Technology 2014

[8] Zhang Xiaoling. Explore the brand development of Hakka culture in Luodai town [J]. Brand Marketing. 2015, 7 
[9] Gu Hao. Folk art class intangible cultural heritage research methodology and value dimension [J]. Decorative.2015, 10

[10] Yue Chunling. The "cultural bias" in the Contemporary design art [D]. Northeast Normal University. 2013 